\section{Assessing the place of nicotine vaporisers in tobacco control}

\author{
Coral Gartner, ${ }^{1}$ Wayne Hall ${ }^{2}$
}

The substantial interest among smokers in e-cigarettes and other nicotine vaporisers has taken the tobacco control field by surprise and produced strongly polarised responses. Proponents see these products as a disruptive technology that promises to accelerate the end of tobacco smoking by increasing quitting and providing a safer longer term alternative to the combustible cigarette. Their opponents see them as a threat to tobacco control that will allow the cigarette industry to subvert tobacco control policies, renormalise smoking and recruit new smokers.

It has been very difficult to assess the plausibility of these radically different scenarios because the evidence on who uses these devices and how they are used has been mostly limited to cross-sectional studies. Much better data from prospective studies are needed to clarify how many smokers are using them to quit and with what success; how many are using them as a full-time substitute for smoking; and how many are only using them when smoking is forbidden but otherwise continuing to smoke (dual use). It has also been unclear to what extent adolescents are using these products. Are those using them using them with or without nicotine? How many simply experiment, without continuing to use, and of those who do continue to use how many become smokers and how many of these would have done so in the absence of e-cigarettes?

The tobacco control field has begun to address the evidence gap by a dramatic increase in research over the past 5 years (see figure 1). The current issue contains a good cross section of the types of studies that are being done.

Better measures of vaping are essential. Because of the low prevalence of vaping in earlier surveys some authors have defined vapers as people who have 'ever used' a nicotine vaporiser or used one in

\footnotetext{
${ }^{1}$ School of Public Health, The University of Queensland, Herston, Queensland, Australia; ${ }^{2}$ Centre for Youth Substance Abuse Research, The University of Queensland, CYSAR K Floor Mental Health Centre, Herston, Queensland, Australia

Correspondence to Dr Coral Gartner, School of Public Health, The University of Queensland, Public Health Building, Herston, Qld 4029, Australia; c. gartner@uq.edu.au
}

the last month. These measures overestimate regular and current use because they include many infrequent users who report using vaping products out of curiosity rather than for quitting, many more of whom will discontinue their use compared to smokers who use them to quit smoking. ${ }^{1}$

It is also essential to collect better data on the type of vaping devices that are used because the vaporiser market has rapidly evolved in recent years. Earlier 'cigalikes' that tried to mimic the look, feel and taste of combustible cigarettes have been supplanted by refillable tank systems favoured by vapers who see vaping as a unique activity (rather than another form of smoking), complete with its own language (eg, 'vaping', 'subohming', 'cloud-chasing'). The move away from cigalikes to tank systems has been motivated by the search for devices that deliver nicotine more efficiently and that allow users to customise their device and the vaping liquid such as adjusting nicotine levels and flavours. Tank systems are now so advanced that experienced users can achieve nicotine doses that rival those from cigarettes under certain conditions. ${ }^{2}$

The flavouring of the e-liquid ${ }^{3} 4$ has also evolved from replicating the tobacco taste of cigarettes to providing an incredible array of non-tobacco flavours. Experimenting with flavours is an integral experience for many vapers. The potential risks from inhaling flavourings, which are often present in e-liquids in concentrations around $1-4 \%,{ }^{4}$ is an emerging area of research concern. Flavouring additives such as vanillin/ethyl vanillin and liquorice have a long history of use in tobacco cigarettes, $^{5}$ where their main health impact has been to increase the palatability of the inhaled smoke. The long-term health effects of inhaling flavours in the absence of the harmful constituents of tobacco smoke has not been fully quantified. Some flavours may be relatively benign, but others, such as diacetyl, raise more concerns. ${ }^{6}$ Further research is needed to identify and eliminate the flavourings that are likely to pose the most risk to vapers.

It is critical to know what smokers think about the benefits and safety of vaporisers. Do they believe them to be useful cessation aids or do they think that vaping could make quitting harder? Are nicotine vaporisers an acceptable and attractive alternative to combustible cigarettes or are they seen as inferior? Rooke et $a l^{7}$ used qualitative interviews and focus groups to explore smokers' and former smokers' perceptions of vaping products, finding a range of divergent views. While some saw vaporisers as a potentially helpful cessation aid, others were sceptical about these devices' benefits and safety, with some ironically preferring the certainty of the harm from smoking cigarettes to the uncertain benefits and risks of vaping. Over-regulation of vaping products can have the perverse effect of denying smokers access to a less harmful option, ${ }^{8}$ while allowing much more dangerous cigarettes to remain freely available. However, underregulation may also prevent smokers who could benefit from switching, from doing so due to low consumer confidence in the quality of the devices and liquids. Finding the optimal regulatory sweet spot between prohibition and regulatory anarchy is a difficult task given the lack of consensus in the tobacco control community about how much regulation is necessary and what aspects of these products should be regulated and how.

The hybrid nature of these products makes them difficult to fit within existing regulatory categories. The result has been divergent approaches to regulation. A vaping product may be regulated as a medical device/medicine for cessation use only, as a tobacco product, a general consumer good, as a non-tobacco recreational substance or as a poison because they contain nicotine (eg, Australia). We should require evidence of efficacy and safety of devices for which therapeutic claims are made. But restricting smokers' access to nicotine vaporisers only when they are approved for use as medicines (as in Australia) may have the unintended effect of conferring a monopoly on products owned by industrial entities with the capital to navigate the pharmaceutical regulatory process, such as the tobacco industry. ${ }^{9}$

The impending Food and Drug Administration deeming regulations in the USA and the Tobacco Products Directive in Europe will affect the types of vaping products on the market. However, regulations over the sale and use of vaping products have already been enacted in many jurisdictions. Some states in the USA ${ }^{10}$ and in Australia, ${ }^{11}$ have included vaping products within the definition of tobacco products in tobacco control legislation, 
Figure 1 Publications per year including e-cigarette related terms. Legend: Results of PubMed Search ( $\left.{ }^{\star} 26 / 02 / 2016\right)$ for (vaping) OR ('electronic cigarette/s) OR ('e-cigarette/ s') OR ('e-cig/s') OR ('electronic nicotine device/s') OR ('electronic nicotine delivery system/s').

thereby applying the same restrictions as apply to sale and use of cigarettes. Others have created vaping product specific regulations and many are yet to decide how to regulate sale and public use. In the absence of licensing vaping product retailers, researching the vaping market will require new research methods to identify these retail outlets, such as the method used by Kim et $a l^{12}$ to compile a list of vape shops by using a combination of online databases and a crowdsourcing Internet marketplace.

Monitoring the activities of the tobacco industry in the vaping product market is and ought to be an active area of inquiry. Products marketed by tobacco companies in the USA have been limited so far to cigalike devices. They comprise previously independent brands (eg, Blu, Green Smoke, Logic) and those developed by these companies (eg, MarkTen, Vuse). ${ }^{13}$ Cigalike devices may be more attractive products for combustible cigarette companies to market rather than refillable tank devices, because advertisements featuring these products ${ }^{14}$ have the potential benefit for the tobacco industry of reglamourising combustible cigarettes due to their similar appearance, which remain their main product.

The debate about vaping has generated almost as much heat and steam as the

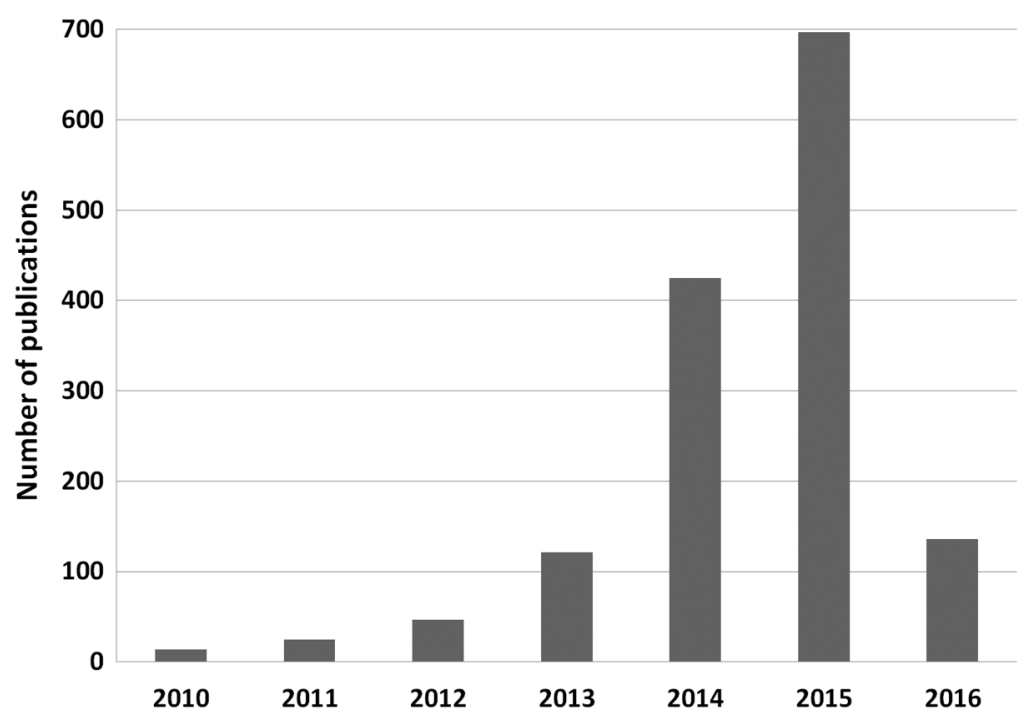

devices themselves. The papers in this issue should generate robust, but, civil and constructive discussion of these important topics. Better research is needed to enable regulators to develop regulatory responses that capture the potential public health benefits of these devices while minimising the potential risks that some ways of promoting their use may pose to public health.

Contributors CG wrote the first draft. WH contributed new text. Both authors revised the manuscript.

Funding National Health and Medical Research Council, 10.13039/501100000925, NHMRC Career Development Fellowship GNT1061978.

Competing interests None declared.

Provenance and peer review Not commissioned; internally peer reviewed.

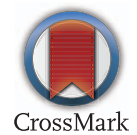

To cite Gartner C, Hall W. Tob Control 2016;25:e1-e2.

Tob Control 2016;25:e1-e2.

doi:10.1136/tobaccocontrol-2016-053025

\section{REFERENCES}

1 Amato MS, Boyle RG, Levy D. How to define e-cigarette prevalence? Finding clues in the use frequency distribution. Tob Control 2016;25:e24-9.

2 Ramôa CP, Hiler MM, Spindle TR, et al. Electronic cigarette nicotine delivery can exceed that of combustible cigarettes: a preliminary report. Tob Control 2016;25:e6-9.

3 Davis B, Razo A, Nothnagel E, et al. Unexpected nicotine in Do-it-Yourself electronic cigarette flavourings. Tob Control 2016;25:e67-8.

4 Tierney PA, Karpinski CD, Brown JE, et al. Flavour chemicals in electronic cigarette fluids. Tob Control 2016:25:e10-5.

5 Boyle P. Tobacco: science, policy and public health. Oxford: OUP, 2010.

6 Sahakian N, Kreiss K. Lung disease in flavoring and food production: learning from butter flavoring. $A d v$ Food Nutr Res 2009;55:163-92.

7 Rooke C, Cunningham-Burley S, Amos A. Smokers' and ex-smokers' understanding of electronic cigarettes: a qualitative study. Tob Control 2016;25: e60-6.

8 Hall W, Gartner C, Forlini C. Ethical issues raised by a ban on the sale of electronic nicotine devices. Addiction 2015:110:1061-7.

9 British American Tobacco e-cigarette wins UK medicine licence. The Guardian 20165 January 2016.

10 Lempert LK, Grana R, Glantz SA. The importance of product definitions in US e-cigarette laws and regulations. Tob Control 2016;25:e44-51.

11 Douglas H, Hall W, Gartner C. E-cigarettes and the law in Australia. Aust Fam Physician 2015:44:415-18.

$12 \operatorname{Kim}$ AE, Loomis $B$, Rhodes $B$, et al. Identifying e-cigarette vape stores: description of an online search methodology. Tob Control 2016;25:e19-23.

13 Seidenberg $A B$, Jo $C L$, Ribisl KM. Differences in the design and sale of e-cigarettes by cigarette manufacturers and non-cigarette manufacturers in the USA. Tob Control 2016;25:e3-5.

14 Cantrell J, Emelle B, Ganz 0, et al. Rapid increase in e-cigarette advertising spending as Altria's MarkTen enters the marketplace. Tob Control 2016;25: e16-8. 